\title{
SU-8 Based Piezoresistive Mechanical Sensor
}

Thaysen, Jacob; Yalcinkaya, Arda Deniz; Vestergaard, R.K.; Jensen, Søren

\section{Published in:}

The Fifteenth IEEE International Conference on Micro Electro Mechanical Systems, 2002.

Link to article, DOI:

10.1109/MEMSYS.2002.984267

Publication date:

2002

Document Version

Publisher's PDF, also known as Version of record

Link back to DTU Orbit

\section{Citation (APA):}

Thaysen, J., Yalcinkaya, A. D., Vestergaard, R. K., \& Jensen, S. (2002). SU-8 Based Piezoresistive Mechanical Sensor. In The Fifteenth IEEE International Conference on Micro Electro Mechanical Systems, 2002. IEEE. https://doi.org/10.1109/MEMSYS.2002.984267

\section{General rights}

Copyright and moral rights for the publications made accessible in the public portal are retained by the authors and/or other copyright owners and it is a condition of accessing publications that users recognise and abide by the legal requirements associated with these rights.

- Users may download and print one copy of any publication from the public portal for the purpose of private study or research.

- You may not further distribute the material or use it for any profit-making activity or commercial gain

- You may freely distribute the URL identifying the publication in the public portal 


\title{
SU-8 BASED PIEZORESISTIVE MECHANICAL SENSOR
}

\author{
J. Thaysen ${ }^{1,2}$, A.D. Yalçinkaya ${ }^{2}$, R.K. Vestergaard ${ }^{2}$, S. Jensen ${ }^{2}$, M.W. Mortensen ${ }^{2}$, P. Vettiger ${ }^{3}$ and \\ A. Menon ${ }^{2}$ \\ ${ }^{1}$ CANTION A/S and ${ }^{2}$ Mikroelektronik Centret, Technical University of Denmark, bldg. 345 east, DK-2800 \\ Kgs. Lyngby, Denmark, ${ }^{3}$ IBM Research Laboratory, CH-8803 Rüschlikon, Switzerland.
}

\begin{abstract}
We present the first SU-8 based piezoresistive mechanical sensor. Conventionally, silicon has been used as a piezoresistive material due to its high gauge factor and thereby high sensitivity to strain changes in a sensor. By using the fact that SU-8 is much softer than silicon and that a gold resistor is easily incorporated in SU-8, we have proven that a SU-8 based cantilever sensor is almost as sensitive to stress changes as the silicon piezoresistive cantilever. We demonstrate the chip fabrication, and characterization with respect to sensitivity, noise and device failure.
\end{abstract}

\section{INTRODUCTION}

The use of the SU- 8 polymer within the MEMS field has been exponentially growing during the last couple of years. The fact that SU-8 is very chemically resistant makes it possible for the use as a component material. Due to its ability of defining layers with thicknesses between $1 \mu \mathrm{m}$ and $1 \mathrm{~mm}$ with high aspect ratio (>20) [1], SU-8 has been a popular and cheap alternative to silicon for the fabrication of passive components. Such components include microchannels, micromolds for electroplating or masters for hot embossing. Passive SU-8 based atomic force microscopy (AFM) cantilevers have also been demonstrated [2].

Since silicon exhibits very good mechanical behaviors and also a very high piezoresistive coefficient, SU-8 has so far never been considered as an alternative as a sensor material with integrated readout. In this paper we present the first SU-8 based sensor with piezoresistive readout which is a cheap and easy fabrication alternative compared to silicon sensors. A cantilever with piezoresistive readout is presented as an example of such a sensor. Cantilevers with piezoresistive readout are normally used as stress sensor, e.g. AFM probes and surface stress sensors for detection of bio-molecular interactions.

\section{THEORY}

In order to illustrate the sensitivity of the SU-based piezoresistive cantilever, it is compared to the sensitivity of a piezoresistive silicon cantilever. In this example the surfaces stress sensitivity is compared for the two different sensors.
When molecules bind to the surface of a cantilever, the surface stress $\sigma_{s}$ changes due to molecular interactions. This stress change can then be picked-up in the integrated piezoresistor. A simple expression for the sensitivity can be obtained by assuming that the cantilever only consists of one material and an infinitely thin resistor is placed on top of the cantilever. The relative change in resistance can be written as:

$\frac{\Delta R}{R} / \sigma_{s}=-K \cdot \frac{4}{h \cdot E}$

where $K$ is the gauge factor, $E$ is Young's modulus and $h$ is the thickness of the cantilever.

In our sensor, a thin gold film is used as the piezoresistor. Gold has a low gauge factor $\left(K_{A u}=2\right)$ compared to silicon $\left(K_{S i}=140\right)$ and is therefore considered inferior to silicon as a piezoresistive sensor material. From equation (1) it is seen that the $K / E$ actually determines the stress sensitivity of the cantilever for the same thickness. Since SU-8 has a Young's modulus of $5 \mathrm{GPa}$ and silicon has a Young's modulus of $180 \mathrm{GPa}$, the ratios becomes $(K / E)_{\mathrm{Si}}=0.8 \mathrm{GPa}^{-1}$ and $(K / E)_{\mathrm{SU}-8 / \mathrm{Au}}=0.4 \mathrm{GPa}^{-1}$, which is only a factor of 2 in sensitivity in favor of silicon. The sensitivity of the SU-8 based piezoresistive cantilever can in principle be enhanced by integrating a piezoresistor material with even higher gauge factor. It is for example possible to integrate a sputtered silicon piezoresistor with a gauge factor of about 20. But in order to use Youngs's modulus for SU-8 in the $K / E$ relation, the stiffness of the piezoresistor should be neglectable compared to the SU-8 cantilever. This can only be obtained by reducing the thickness of the poly silicon resistor which increases the noise significantly and thereby reducing the signal to noise ratio.



Figure 1: Schematic of the chip design.

\footnotetext{
${ }^{1}$ Jacob Thaysen, CANTION A/S, DTU, CAT, bldg. 347, DK-2800 Lyngby, email: jt@ cantion.com, tlf. +45 45256409
} 


\section{DESIGN}

The design of the cantilever chip is shown in figure 1. As can be seen from the figure that the chip consists of two cantilevers with integrated resistors and two resistors on the substrate forming a whole Wheatstone bridge. The advantage of this design is that one of the cantilevers can be used as a measurement cantilever, while the other is used as common-mode rejection filter. The parameters for the fabricated cantilevers are shown in table 1.

\section{Table 1: Design parameter:}

\begin{tabular}{|l|l|l|}
\hline Parameter & Value & Unit \\
\hline Cantilever length & 200 & $\mu \mathrm{m}$ \\
Cantilever width & 100 & $\mu \mathrm{m}$ \\
Cantilever Thickness & 7.3 & $\mu \mathrm{m}$ \\
Spring constant & 7 & $\mathrm{~N} / \mathrm{m}$ \\
Resonant frequency & 49 & $\mathrm{kHz}$ \\
\hline
\end{tabular}

Optical pictures of the fabricated chip can be seen in figure 2 . In figure $2 \mathrm{a}$, the two cantilevers can be seen. Figure $2 b$ shows a close-up of one of the cantilevers. The meander-like resistor structure is clearly seen in the picture.
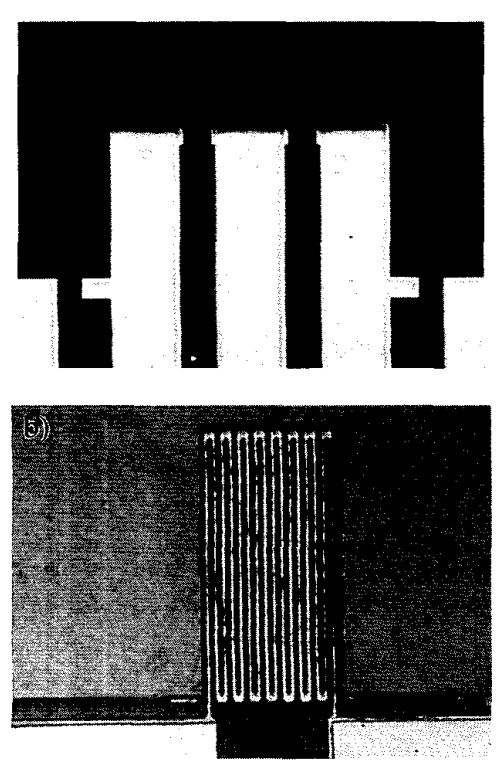

Figure 2: Optical images of the chip. Image a) shows the two cantilevers inserted in the on-chip Wheatstone bridge, while image b) shows a close-up of the cantilever with integrated meander-type resistor.

\section{FABRICATION}

The SU-8 based cantilever with integrated piezoresistive readout is fabricated on a silicon substrate, which is only used in order to be able to handle the chips during the processing. First, a $\mathrm{Cr} / \mathrm{Au} / \mathrm{Cr}$ layer is deposited on the silicon wafer see figure $3 \mathrm{a}$. This is used as a very fast etching sacrificial layer, developed by G. Genolet [3]. A thin layer of SU-8 is spun on the wafer and patterned as upper cantilever layer (figure $3 b$ ). A $1 \mu \mathrm{m}$ thick gold layer is deposited on top of the wafer. The conductor mask is transferred to the wafer by standard photoresist/photolithography and the conductors are etched (figure 3c). A $400 \AA$ thick gold layer is deposited and the resistors are defined by the same procedure as above (figure $3 \mathrm{~d}$ ). The metal is completely encapsulated in SU- 8 by depositing and patterning of a $5.8 \mu \mathrm{m}$ thick SU-8 layer for the lower SU-8 cantilever layer (figure 3e). A $350 \mu \mathrm{m}$ thick SU-8 layer is spun on the wafer an patterned as the chip substrate (figure $3 \mathrm{f}$ ). The chip is finally released by etching of the sacrificial layer (figure $3 g$ ).

(a)

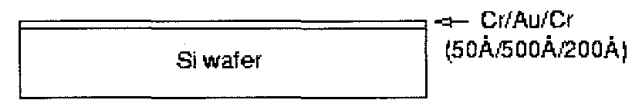

(b)

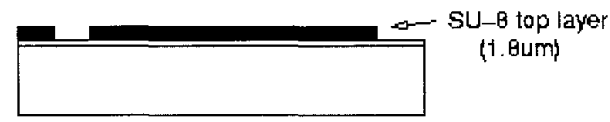

(c)

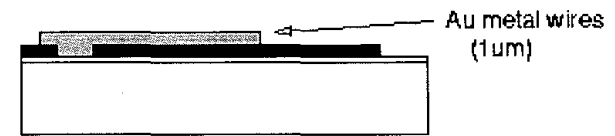

(d)



(e)

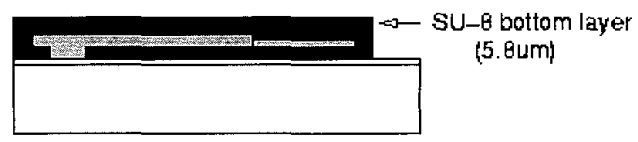

$(t+)$



(g)

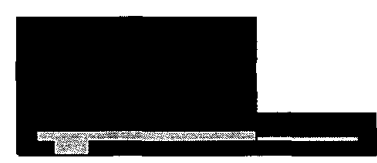

Release of chips

Figure 3: Process sequence for the fabrication of the SU-8 cantilever.

\section{CHARACTERIZATION}

SEM micrograph of the finished chip is seen in figure 4. Figure 4a shows the cantilevers seen from below. The cantilevers are seen to be completely straight. Figure $4 b$ shows the cantilevers seen from above. It is not possible to see the resistors or the conductors on this micrograph. 

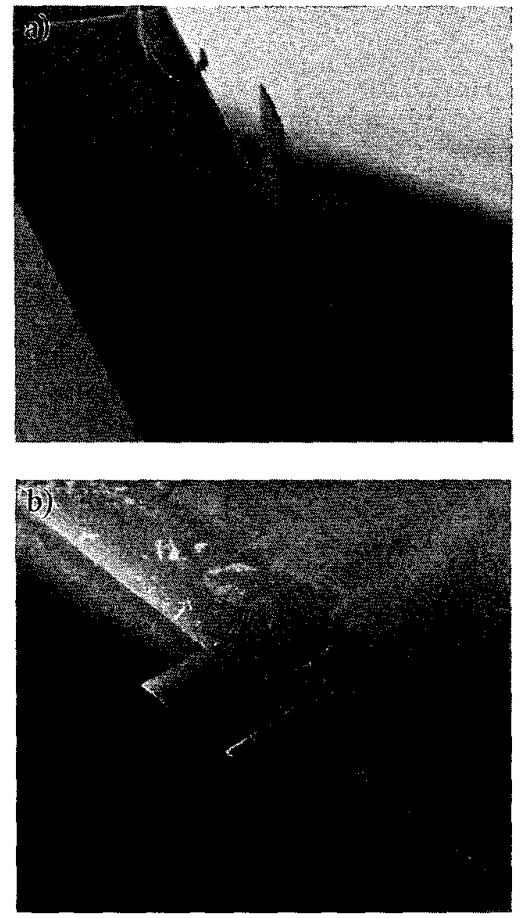

Figure 4: SEM micrographs of the cantilever chip, a) seen from below and $b$ ) seen from the top.

The deflection sensitivity of the piezoresistive SU-8 cantilevers has been measured by observing the relative change in resistance as a function of the cantilever deflection. The result is shown in figure 5. It is seen that a straight line can be obtained from the measurement. This states that the deformation is purely elastic.

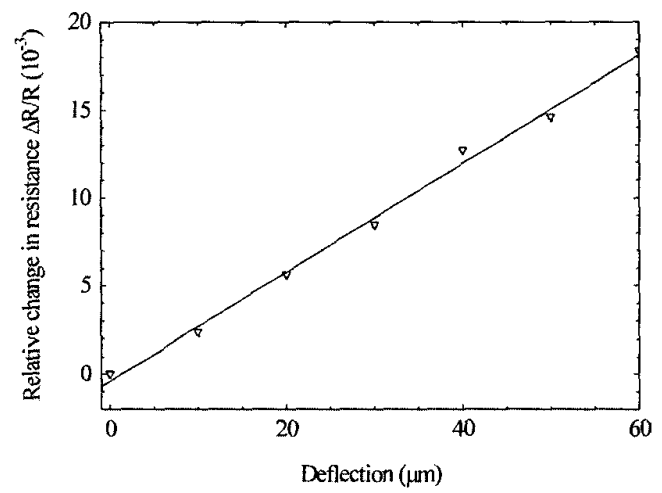

Figure 5: Deflection sensitivity measurement graph

The deflection sensitivity can be found from the slope of the straight line to $\frac{\Delta R}{R} / z=0.3 \mathrm{ppm} / \mathrm{nm}$, which yields a gauge factor of $K=4$. The reason that the gauge factor is higher than expected is probably due to different behavior of the very thin gold resistor than a bulk gold resistor.

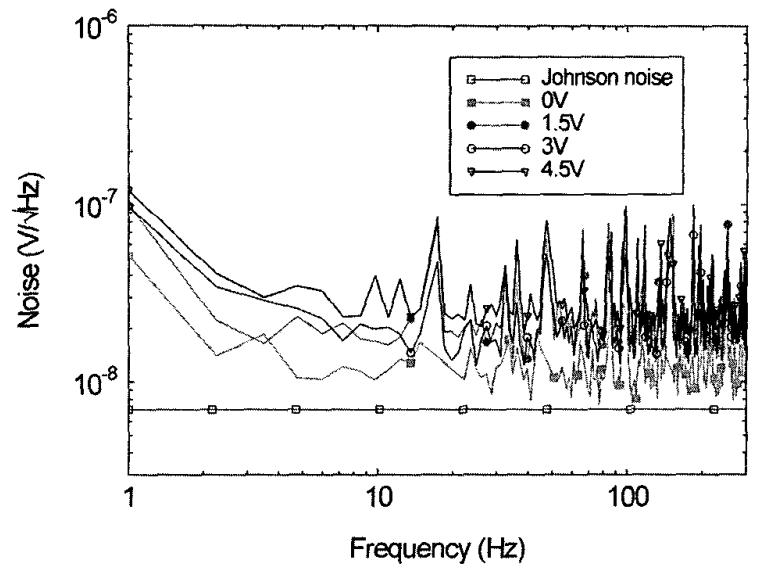

Figure 6: The graph shows the electrical noise as a function of frequency for different supply voltage.

The minimum detectable deflection or minimum detectable surface stress is defined by the noise in the system. Since the vibrational noise is considerably lower than the electrical noise sources in this resistor setup, only the Johnson noise and the $1 / \mathrm{f}$ noise is considered here. In figure 6 , the noise is measured as a function of frequency for different input voltages. It is seen from the graph that the 1/f noise is very low with a knee frequency of about 10 $\mathrm{Hz}$ for a Wheatstone bridge supply voltage of $4.5 \mathrm{~V}$. It is seen from the graph the measured Johnson noise floor is about a factor of 2 higher than expected.

Table 2: Performance of the SU-8 based piezoresistive cantilever compared to a piezoresistive silicon cantilever.

\begin{tabular}{|c|c|c|}
\hline Parameter & $\begin{array}{c}\text { SU-8 } \\
\text { cantilever }\end{array}$ & $\begin{array}{l}\text { Si cantilever } \\
\text { (optimized) }\end{array}$ \\
\hline $\begin{array}{c}\text { Deflection sensitivity } \\
{\left[\mathrm{nm}^{-1}\right.}\end{array}$ & $0.3 \cdot 10^{-6}$ & $4.8 \cdot 10^{-6}$ \\
\hline $\begin{array}{c}\text { Minimum detectable } \\
\text { deflection }[\AA]\end{array}$ & 4 & 0.4 \\
\hline $\begin{array}{c}\text { Surface stress } \\
\text { sensitivity }[\mathrm{N} / \mathrm{m}]^{-1}\end{array}$ & $3 \cdot 10^{-4}$ & $1 \cdot 10^{-3}$ \\
\hline $\begin{array}{l}\text { Minimum detectable } \\
\text { surface stress }[\mathrm{N} / \mathrm{m}]\end{array}$ & $1 \cdot 10^{-4}$ & $2 \cdot 10^{-5}$ \\
\hline
\end{tabular}

From the above measurements it is possible to list the performance of the SU- 8 based piezoresistive cantilever. The performance is listed in table 2 with respect to deflection sensitivity, minimum detectable deflection, surface stress sensitivity and minimum detectable surface stress. Furthermore, the performance is compared to an optimized silicon piezoresistive cantilever. It is seen from the table that the minimum detectable deflection is 10 times better for the silicon cantilever, but only 5 times better for the minimum detectable surface stress, nevertheless the SU-8 based piezoresistive cantilever can be used as a surface stress bio-chemical sensor, since the change in surface stress due to molecular 
interactions on a cantilever surface is normally in the order of $10^{-3}-1 \mathrm{~N} / \mathrm{m}[4,5]$. Reducing the thickness of the cantilever can increase the surface stress performance even further. As seen from equation 1, the sensitivity is inversely proportional with the thickness. With the given technology it is possible to decrease the cantilever thickness a factor of 2 and thereby decrease the minimum detectable surface stress with a factor of 2 .

Electron migration of the gold resistor has turned out to limit the supply voltage. As seen from equation 2 the mean time to failure $(M T F)$ due to electron migration decreases as the square of the current density $(j)$.

$M T F \propto \frac{1}{j^{2}} \exp \left(Q / k_{B} T\right)$

where $Q$ is the diffusion activation energy and $T$ is the temperature. Since the gold resistor has a very small crosssectional area this effect quickly becomes dominant factor for the working of the device.

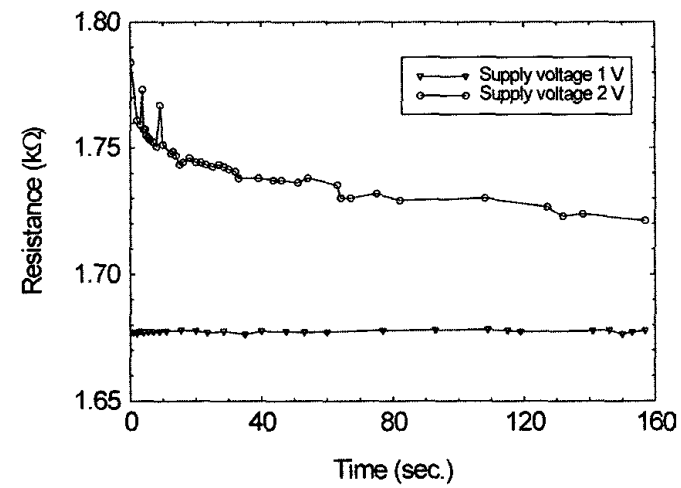

Figure 7: The graph shows the resistor value becomes unstable for a supply voltage more than $1 \mathrm{~V}$ over the resistor.
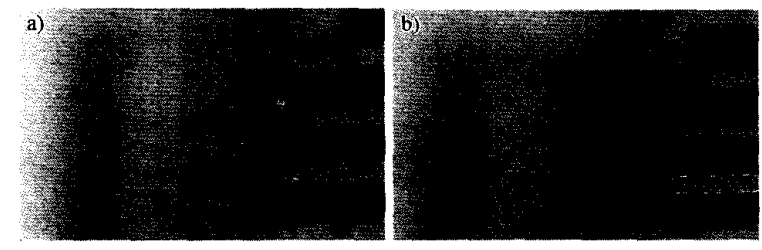

Figure 8: In both images the lower cantilever is exposed to a voltage drop. In a) the cantilever is exposed to $1 \mathrm{~V}$ and no plastic deformation is obtained and b) the cantilever is exposed to $2 \mathrm{~V}$ at which it is deformed.

Figure 7 shows two plot of the change in resistance value as a function of time for different voltage drops over a gold resistor. The resistor value becomes unstable for supply voltages more than $1 \mathrm{~V}$. As seen in the figure, the resistor value for a voltage drop of $2 \mathrm{~V}$ is unstable due to the deformation of the cantilever. After the deformation is completed the resistor value stabilizes. For supply voltages higher than $3 \mathrm{~V}$ the resistor burns within a few seconds. The reason for the instability of the resistance is not only due to electron migration, but also due to a plastic deformation of the cantilever. Figure 8 shows the plastic deformation of the cantilever for different supply voltages. It can be seen that no deformation is obtained for a supply voltage of $1 \mathrm{~V}$ while the cantilever is severely bended due to a plastic deformation for a supply voltage of $2 \mathrm{~V}$. The reason for the plastic deformation is probably due a significant selfheating of the resistor. Since the SU- 8 polymer is a very poor heat conductor, the resistor self-heating has probably heated the SU-8 non-uniformly through the cantilever, which has lead to the bending of the cantilever. From this experiment it can be concluded that the maximum voltage drop over the cantilever resistor should be $1 \mathrm{~V}$, corresponding to a Wheatstone bridge supply voltage of 2 $\mathrm{V}$.

\section{CONCLUSIONS}

We have demonstrated the design, fabrication and performance of the first SU-8 based piezoresistive cantilever. The fabrication of the sensor is very cheap and easy compared to conventional silicon fabrication technology, and the use of SU-8 opens up for a whole new design space. The theory described why the surface stress sensitive cantilever potentially is as sensitive as a silicon cantilever, and the characterization of the fabricated cantilever supports this theory. The measured gauge factor was a factor of 2 higher than expected. For the current design, the minimum detectable surface stress is still a factor of 5 higher than for the optimized silicon cantilever, but is still sensitive enough for the use in many biochemical sensor applications.

\section{ACKNOWLEDGEMENT}

We would like to thank Ole Hansen and Anja Boisen from Mikroelektronik Centret for fruitful discussions and many insightful inputs.

\section{REFERENCES}

[1] H. Lorenz et al.. "SU-8: a low-cost negative resist for MEMS". J. Micromech. Microeng., 7: pp.121-124, 1997.

[2] G. Genolet et al., "Soft, entirely photoplastic probes for scanning force microscopy", Review of Scientific Instruments, Vol. 70 (5),pp. 2398-401,1999.

[3] G. Genolet, "New photoplastic fabrication techniques and devices based on high aspect ratio photoresist", Ph.d. thesis from Swiss Federal Institute of Technology Lausanne, 2001.

[4] G. Wu et al., "Origin of nanomechanical cantilever motion generated from biomolecular interactions", Proceedings of the National Academy of Sciences of the USA, pp.1560-1564, 2001.

[5] J. Thaysen et al., "A microcantilever-based detection principle for $\mu \mathrm{TAS}$ ", Proceedings of $\mu$ TAS 2001, Monterey, USA, pp. 447-449, 2001. 